\title{
Primary Adrenal Glucocorticoid Excess
}

National Cancer Institute

\section{Source}

National Cancer Institute. Primary Adrenal Glucocorticoid Excess. NCI Thesaurus. Code C127158.

Supranormal glucocorticoid concentrations resulting from a condition originating within the adrenal gland. 